\title{
Chemical and electrochemical synthesis of crosslinked aniline sulfide resin
}

\author{
Seyed Hossein Hosseini ${ }^{1}$, Maryam Malekdar ${ }^{2}$ and Shaghayegh Naghdi ${ }^{3}$
}

A strategy was used to control the crosslinking of polyaniline (PANi) to allow the synthesis of high-molecular-weight, conductive copolymers. Aniline sulfide resin (ASR) was synthesized by the reaction of aniline and sulfide dichloride. Crosslinked ASR (ASC) was prepared in the presence of ammonium peroxydisulfate and aniline in an aqueous solution as the oxidant and monomer, respectively. The graft copolymer was obtained by oxidizing aniline on ASR in the presence of an initiator and an excess amount of aniline, in which the aniline was oxidized to form PANi through a radical cation mechanism. By changing the ratio of ASR to aniline during the polymerization reaction, it was possible to control the number of crosslinked sites, the electrical conductivity, the molecular weight and the solubility. The structure was characterized by spectroscopic data. The method introduced here describes a strategy for controlling the molecular weight of PANi and in turn its physical properties. This approach may be extended to the synthesis and modification of other polymers. Electropolymerization was carried out by coating ASR on the surface of a glassy carbon disk electrode and then growing this on graft copolymer ASC in the presence of fresh aniline and an acidic solution. The electrical conductivity of ASC was studied by a four-point probe method and produced a conductivity of $8.5 \times 10^{-3} \mathrm{~S} \mathrm{~cm}^{-1}$. Polymer Journal (2010) 42, 640-647; doi:10.1038/pj.2010.56; published online 7 July 2010

Keywords: ASR; conducting polymers; electropolymerization

\section{INTRODUCTION}

Nitrogen- and sulfide-containing polymers have an important role in electrically conductive polymers, such as polyaniline (PANi), polypyrrole, polythiophene and poly(phenylene sulfide) (PPS).$^{1-3}$ PPS and PANi are heteroatom-containing polymers with unique properties. Although PPS provides a good level of chemical and thermal stability, ${ }^{4}$ PANi is important because of its ability to form electrically conductive films. ${ }^{5}$

Among these conductive polymers, PANi has important technological applications in many areas, owing to its excellent electrical properties, good environmental stability, facile synthesis and low monomer cost. ${ }^{6,7}$ To the best of our knowledge, although many efforts have been made to investigate its synthesis, structure, properties and applications, PANi synthesized by chemical or electrochemical polymerization, usually exhibits an ill-defined structure. Nevertheless, there are still some unsolved problems with regard to its structure and properties because of the complexity of its molecular structure and the poor solubility of its common organic solvents, which to some extent limit investigations of the structure-property relationship and the conducting mechanism. It should be noted out that the challenging aspect of synthesis research is the design of new PANi derivatives with fixed conjugation lengths as the model polymers for identifying the conducting mechanism of common PANi. In addition to a low mechanical strength, the adhesion of PANi on solid substrates is another important factor that has limited its utility in applications such as in antistatic coatings. ${ }^{8,9}$ For example, although PANi films cast from $N$-methyl-2-pyrrolidone (NMP), dimethyl sulfoxide (DMSO) or dimethylformamide solutions adhere strongly to glass in dry atmospheres, adhesion quickly fails when the films are immersed in water. Increasing the molecular weight of PANi is expected to increase the strength of its adhesion as well as its mechanical strength. The effect of aniline formaldehyde resin on the conjugation length and structure of doped PANi was investigated by Ho et al. ${ }^{10,11}$ by spectral and thermal studies. Kumar et al. ${ }^{12}$ synthesized aniline formaldehyde condensate coated on silica gel for the removal of hexavalent chromium.

The method described herein uses aniline sulfide resin (ASR) to crosslinked ASR (ASC). The copolymer can be synthesized by oxidizing ASR-aniline mixtures at room temperature. A wide range of molecular weights can be obtained by simply adjusting the ratio of ASR to aniline before polymerization. The conductivity and solubility of the copolymer are a function of the ratio of ASR to aniline and therefore can be varied systematically. The use of this method for copolymer preparation results in significantly better adhesion on glass both in air and water compared with pure PANi.

${ }^{1}$ Department of Chemistry, Faculty of Science, Islamic Azad University, Islamshahr Branch, Tehran, Iran; ${ }^{2}$ Department of Chemistry, Faculty of Technical and Engineering Azad University, Shahr-e-Ray Unit, Tehran, Iran and ${ }^{3}$ Department of Organic Chemistry, Faculty of Chemistry, Tarbiat Moallem University, Tehran, Iran 


\section{EXPERIMENTAL PROCEDURE}

Materials

Aniline monomer (reagent grade, Merck, Darmstadt, Germany) was distilled under reduced pressure and stored below $0{ }^{\circ} \mathrm{C}$. All the other reagents were of analytical grade and were used without further purification, including ammonium peroxydisulfate, sulfide dichloride, sodium hydrate $(\mathrm{NaOH}), \mathrm{HCl}(37 \%)$, hydrogen peroxide $\left(\mathrm{H}_{2} \mathrm{O}_{2}, 30 \%\right)$ and ethanol. Water was double distilled and bubbled for 30 min with $\mathrm{N}_{2}$.

\section{Physical measurements}

Conductivity changes were measured with a four-probe device (ASTM Standards, F 43-93). ${ }^{1} \mathrm{H}$ and ${ }^{13} \mathrm{C}$ nuclear magnetic resonance $\left({ }^{13} \mathrm{C}-\mathrm{NMR}\right)$ spectra were recorded on a BRUKER 250 NMR spectrometer (Bruker Biospin GmbH, Rheinstetten, Germany) at $400 \mathrm{MHz}$ in deuterated dimethylsulfoxide- $\mathrm{d}_{6}$ with TMS as an internal standard. NMR data are reported in the following order: chemical shift (p.p.m.), spin multiplicity (s, singlet; d, doublet; t, triplet; q, quartet; $\mathrm{m}$, multiplet) and integration. Molecular weights were measured at $30^{\circ} \mathrm{C}$ with gel permeation chromatography (Waters Associates, Milford, MA, USA, model 150-C). Three styragel-packed columns with different pore sizes $\left(10^{4}-10^{6} \mathrm{~A}^{0}\right)$ were used. The mobile phase was NMP with a flow rate of $1.5 \mathrm{ml} \mathrm{min}^{-1}$. The solution concentration was $0.2 \mathrm{wt} \%$. Calibration of the instrument was performed with nine standard samples of monodispersed polystyrene having molecular weights between $3.0 \times 10^{3}$ and $1.4 \times 10^{6}$. The PHILIPS XL30 (Philips, Eindhoven, Netherlands) was used for scanning electron microscopy (SEM). A Fourier-transformed infrared (FT-IR) spectrometer (8101 M-Shimadzu; Shimadzu, Kyoto, Japan) and BRUKER-IF-66.5 were used in spectral measurements of the polymer and were reported as sharp (sh), weak (w), broad (b), middle (m) and shoulder (s). FT-IR spectra were recorded on the 8101-M-Shimadzu and BRUKER-IF-66.5 spectrometers. The vibrational transition frequencies are reported in wave number $\left(\mathrm{cm}^{-1}\right)$. The ultraviolet (UV)-visible spectra were obtained using a UV-visible recording spectrophotometer (Perkin-Elmer Lambda 15; PerkinElmer, Waltham, MA, USA).

\section{Synthesis of ASR}

ASR was synthesized by reacting sulfide dichloride with aniline. In a $100-\mathrm{ml}$ three-necked flask equipped with a stirrer, a condenser and an inlet of nitrogen, $0.2 \mathrm{ml}$ sulfide dichloride in $5 \mathrm{ml}$ diethylether was added dropwise to a mixture of $0.5 \mathrm{~g}$ aniline and $0.1 \mathrm{ml}$ pyridine in $10 \mathrm{ml}$ diethylether. Condensation was carried at $25^{\circ} \mathrm{C}$ for $1 \mathrm{~h}$. The resin was filtered and washed with warm water three times. The resin was kept at $80^{\circ} \mathrm{C}$ under a reduced pressure of $10 \mathrm{kPa}$ for $1 \mathrm{~h}$ to remove any unreacted aniline or sulfide dichloride.

UV ( $m$-cresol): $\lambda_{\max }=220 \mathrm{~nm}$ ( 3.1 intensity), $270 \mathrm{~nm}$ ( 0.7 intensity).

FT-IR: $3490(\mathrm{~m}), 3357(\mathrm{sh})(\mathrm{N}-\mathrm{H}$ stretching), 3034(m)(C-H aromatic stretching), 2925(m) (C-H aliphatic stretching), 1612(sh) (C=S stretching), 1495 (sh)(C=C stretching), 1277(sh), 1169(m)(C-S stretching), 1029(m), 883(m) and 755(sh), 690(m), 496(sh) $\mathrm{cm}^{-1}$.

${ }^{1} \mathrm{H}-\mathrm{NMR}$ (d $\mathrm{d}^{6}$-DMSO) $\delta 2.4$ (DMSO), 6.5-7.5 ( $\mathrm{NH}_{2}$ protons), 8-9 (aromatic protons) p.p.m.

${ }^{13} \mathrm{C}-\mathrm{NMR}\left(\mathrm{d}^{6}\right.$-DMSO) $\delta 38-40$ (DMSO), 123,127,129,132,142, 145 (aromatic carbons) p.p.m.

\section{Synthesis of PANi-sulfide crosslinked copolymer (ASC)}

In a 150-ml reaction vessel, ASC and aniline monomer were dissolved in $50 \mathrm{ml}$ of $1 \mathrm{M} \mathrm{HCl}$ aqueous solutions with varying ratios. A trace amount of $\mathrm{FeSO}_{4}$ was added to the solutions as a catalyst. Then, $3 \mathrm{ml}$ of $30 \% \mathrm{H}_{2} \mathrm{O}_{2}$ was added dropwise to the solution with vigorous stirring. After polymerization for $6 \mathrm{~h}$ at $25^{\circ} \mathrm{C}$, a dark polymer powder was obtained. The powder was washed with $1 \mathrm{M}$ $\mathrm{HCl}$ until the washing solution became clear. Lower-molecular-weight oligomers were removed by further washing with copious amounts of acetonitrile. The doped (or conducting) forms of the polymers were obtained by acidification of the polymers with $1 \mathrm{M} \mathrm{HCl}$. The base forms of the polymers were obtained by treating the polymers with aqueous $1 \mathrm{M} \mathrm{NH}_{3} \mathrm{OH}$.

UV ( $m$-cresol): $\lambda_{\max }=228 \mathrm{~nm}$ ( 2.3 intensity), $260 \mathrm{~nm} \mathrm{(0.5} \mathrm{intensity),}$ $560-750 \mathrm{~nm}$ (0.2 intensity).

FT-IR: $3400-3600(\mathrm{w})\left(\mathrm{NH}_{2}\right.$ stretching $), 3041(\mathrm{~m}), 1622(\mathrm{~m})(\mathrm{C}=\mathrm{S}$ stretching $)$, 1467 (sh)(C=C stretching), 1090(s)(C-S stretching), 680(m), 750(sh) $\mathrm{cm}^{-1}$.
${ }^{1} \mathrm{H}-\mathrm{NMR}$ (d ${ }^{6}$-DMSO) $\delta 2.4$ (DMSO), 7.3-9 (aromatic protons) p.p.m.

${ }^{13} \mathrm{C}-\mathrm{NMR}$ (d6-DMSO) $\delta$ 38-40 (DMSO), 121, 126, 129, 131, 134, 141, 142, 146 p.p.m

\section{Electrochemical synthesis of ASC}

Electrochemical synthesis of ASC was carried out using a conventional threeelectrode system containing an $\mathrm{Ag} / \mathrm{AgCl}$ reference electrode, a platinum wire counter electrode and a glassy carbon (GC) disk working electrode. The cyclic voltammetric solution was $0.1 \mathrm{M} \mathrm{H}_{2} \mathrm{SO}_{4}$ and $1 \mathrm{M} \mathrm{HCl}(50 / 50 \mathrm{v} / \mathrm{v})$ as the supporting electrolyte. First, ASR was coated as a suitably thin layer (50$60 \mu \mathrm{m}$ ) on the GC disk electrode using a casting method. A cyclic voltammogram of ASR on a GC electrode under the above-mentioned conditions with a $50 \mathrm{mV} \mathrm{s}^{-1}$ scan rate was created. Then, $0.1 \mathrm{M}$ aniline was added to the electrolyte. The graft copolymer was prepared on the GC disk electrode under the same conditions. The scan potential was in the range of -0.1 to $1.5 \mathrm{~V} \mathrm{vs} \mathrm{Ag/}$ $\mathrm{AgCl}$ (11 cycles), and the scan rate was $50 \mathrm{mV} \mathrm{s}^{-1}$. Finally, a blank cyclic voltammogram of ASC on a GC electrode under the same conditions was prepared.

\section{RESULTS AND DISCUSSION}

\section{Spectroscopic studies}

Conductive polymers have a conjugated system of double bonds in a backbone polymer. These polymers have some conventional transfers in the UV region, such as $n \rightarrow \pi^{*}, \pi \rightarrow \pi^{*}$ and so on. The $\pi \rightarrow \pi^{*}$ transfers of conjugated double bonds are related to near-visible regions and are associated to the polaron and bipolaron status, as well as the solution of conductive polymers.

UV-visible spectrum data for ASR and ASC in an $m$-cresol solvent are shown in Figure 1. The above-mentioned polymer (with the aniline pattern) should have two peaks at $221 \mathrm{~nm}$ with a 3.1 intensity and $270 \mathrm{~nm}$ with a 0.7 intensity. Transfer of the first one could be for $n \rightarrow \pi^{*}$ and that of the second one could be for $\pi \rightarrow \pi^{*}$ of a benzoic system. As we know, with an increase in the length of the chain and the subsequent increase in conjugated double bonds, a decrease in the energy $\pi \rightarrow \pi^{*}$ occurs, which causes an increase in the wavelength. The UV-visible spectrum for aniline formaldehyde condensation shows four peaks in $225,280,325$ and $520-720 \mathrm{~nm}$. The peaks at 225 and $280 \mathrm{~nm}$ related to $n \rightarrow \pi^{\star}$ and $\pi \rightarrow \pi^{\star}$ transfers for the benzoic form. The peaks at 325 and $520-720 \mathrm{~nm}$ are related to ASC structure. Obviously, after grafting aniline on ASR, a the wide peak at 560$750 \mathrm{~nm}$ comes from the transfer of the $\pi \rightarrow \pi^{\star}$ quinion of the aniline groups (for the polaron and bipolaron transfers). We also observed more conductivity, which could be due to the couple length of the dual grafts in the system length. As is shown in Figure 1, the polaron and bipolaron transfers are increased by increasing the percentage of

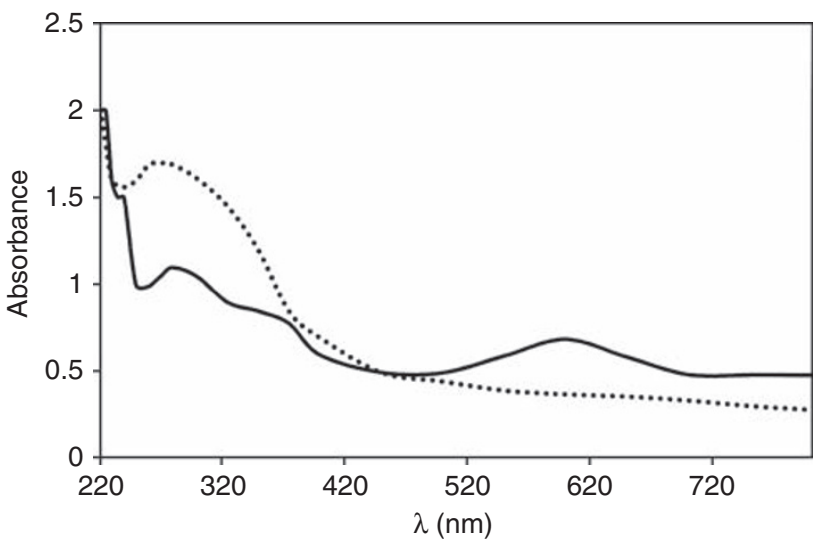

Figure 1 UV-visible spectra of ASR (...) and ASC (-). 


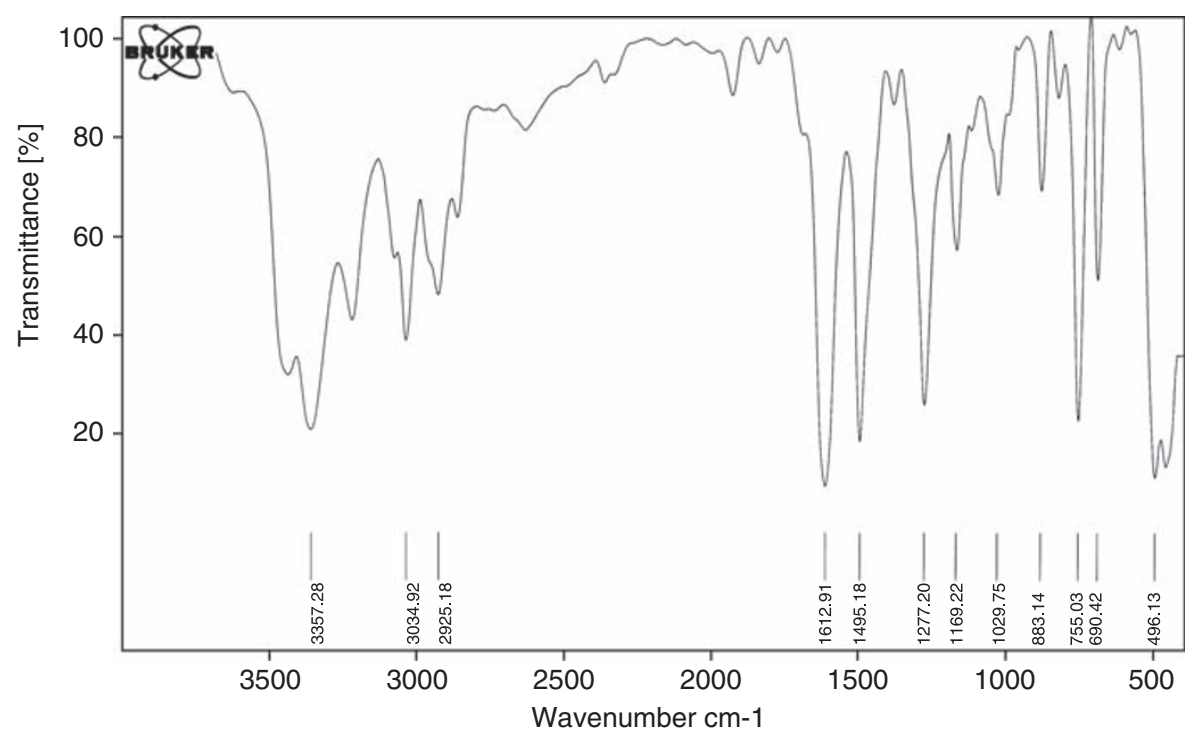

Figure 2 FTIR spectrum of ASR.

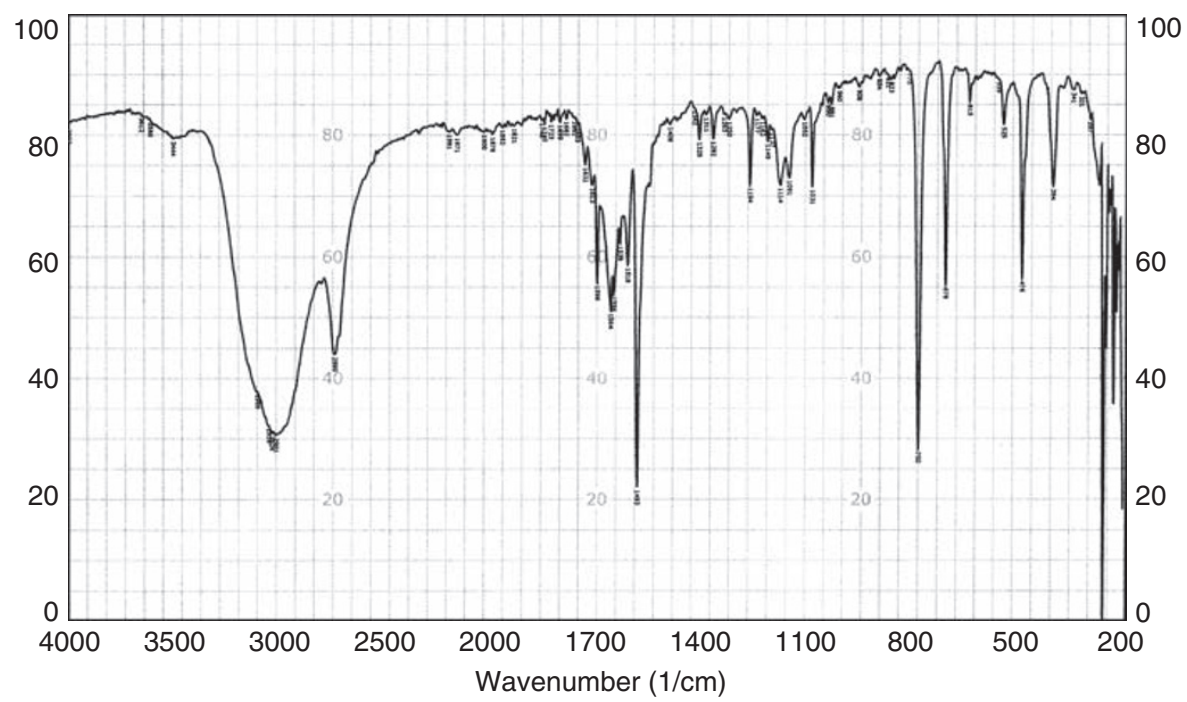

Figure 3 FTIR spectrum of ASC.

aniline in the copolymers, as the conjugated double bond systems were increased.

The FTIR spectra of the ASR and ASC polymers are shown in Figures 2 and 3, respectively. The characteristic absorption bands of ASR are $1612 \mathrm{~cm}^{-1}(\mathrm{C}=\mathrm{S}), 1495 \mathrm{~cm}^{-1}(\mathrm{C}=\mathrm{C})$ and $1169 \mathrm{~cm}^{-1}(\mathrm{C}-\mathrm{S})$, while the absorption peaks of both shifted and decreased ASC are 1622,1467 and $1090 \mathrm{~cm}^{-1}$. This is possibly due to steric interactions and the high molecular weight, which could reduce the energy level interval of the phenyl ring.

The ${ }^{1} \mathrm{H}-\mathrm{NMR}$ spectra of ASR and ASC polymers are illustrated in Figures 4 and 5, respectively. The ${ }^{1} \mathrm{H}-\mathrm{NMR}$ spectrum of ASR shows just one broad peak each for the amino proton at $\delta=7.0-7.7$ p.p.m. and for the aromatic protons at $\delta=8-9$ p.p.m. Notably, the ${ }^{1} \mathrm{H}-\mathrm{NMR}$ amino proton signal of the shifted ASC is $10-11$ p.p.m., which is also possibly due to steric interactions and the high molecular weight. The ${ }^{13} \mathrm{C}-\mathrm{NMR}$ spectrum of ASR is shown in Figure 6. The structure of ASR is confirmed by this ${ }^{13} \mathrm{C}-\mathrm{NMR}$ spectroscopy as well, in which only aromatic carbons at $123-145$ p.p.m. are observed.

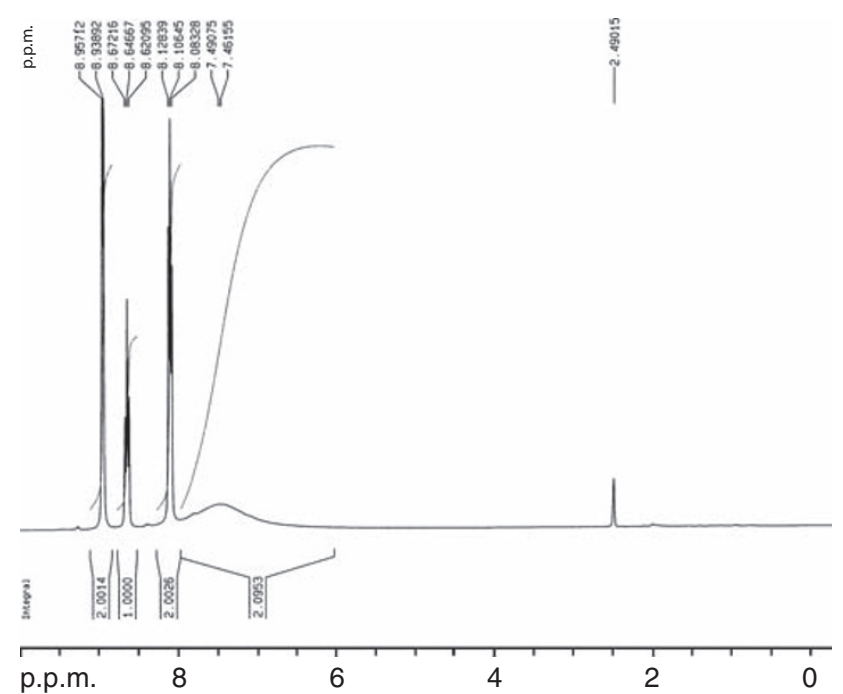

Figure $4 \mathrm{FT}^{1} \mathrm{H}-\mathrm{NMR}$ spectrum of ASR. 




Figure $5 \mathrm{FT}^{1} \mathrm{H}-\mathrm{NMR}$ spectrum of ASC.

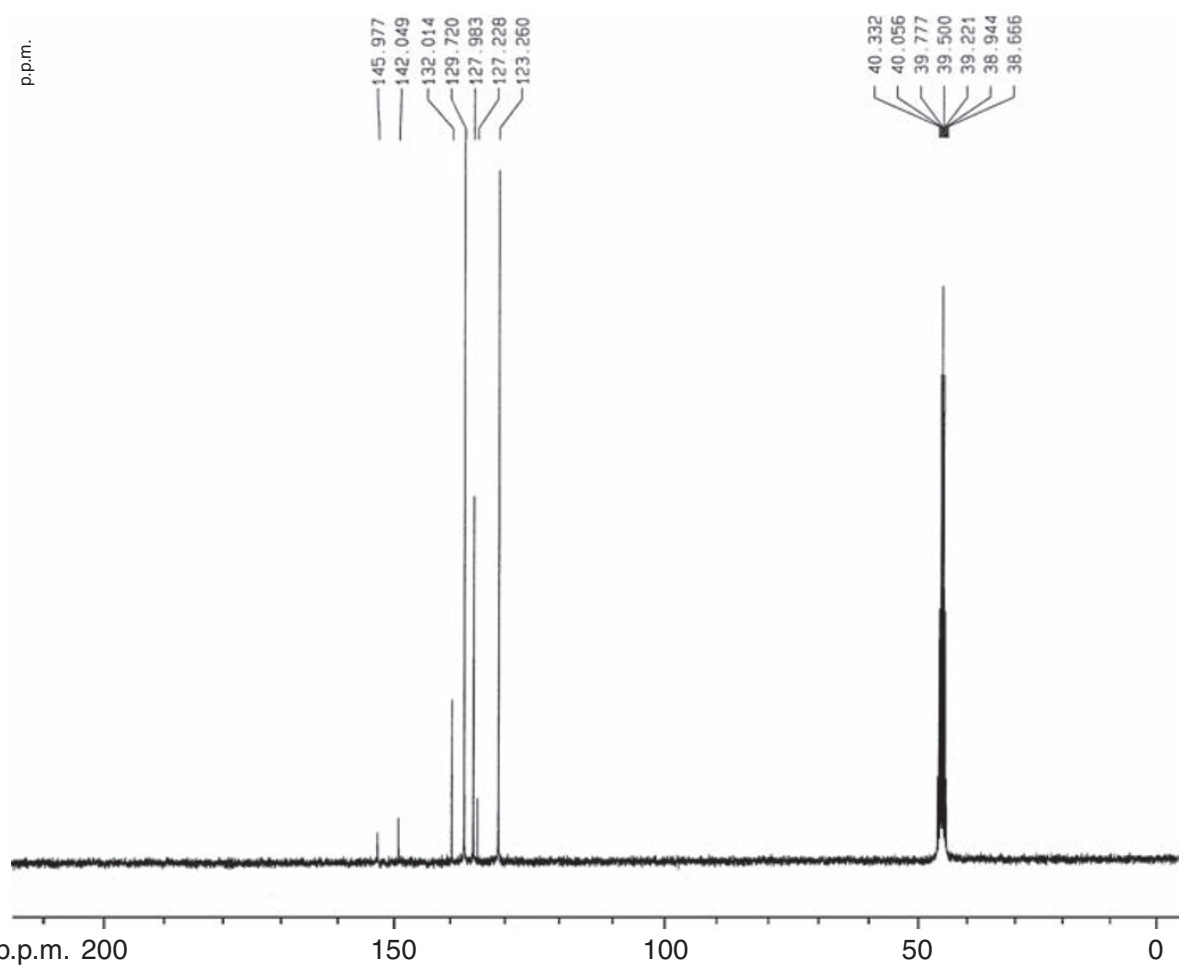

Figure $6 \mathrm{FT}^{13} \mathrm{C}-\mathrm{NMR}$ spectrum of $\mathrm{ASR}$.

\section{Polymerization and kinetics}

ASR can be synthesized as shown in Scheme 1, where the resulting polymer has alternatively substituted aniline and sulfide units. The existing aniline in ASR can be oxidatively polymerized by ammonium peroxydisulfate. The resulting graft copolymer includes both ASR and PANi.

The graft copolymerization occurs readily, although the reaction has steric interactions. Thus, aniline sulfide crosslinked (ASC) copolymer 


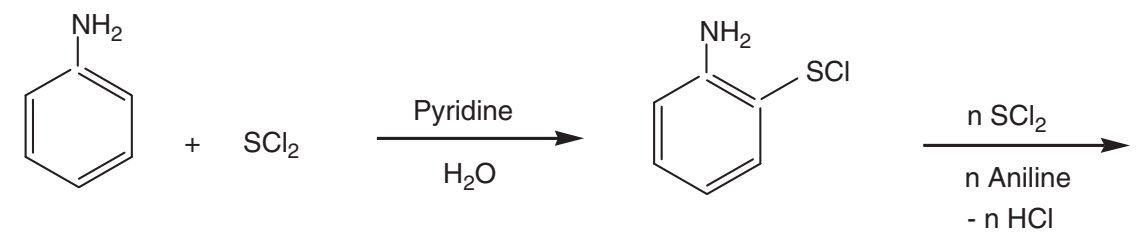<smiles>Cc1cccc(Sc2cccc(Sc3cccc(Sc4cccc(Cl)c4N)c3N)c2N)c1N</smiles>

Scheme 1 Synthesis of ASR.<smiles>CC(C)(C)Sc1cccc(Sc2cccc(C(C)(C)[n+]3ccccc3)c2N)c1N</smiles>

Aniline

ASR
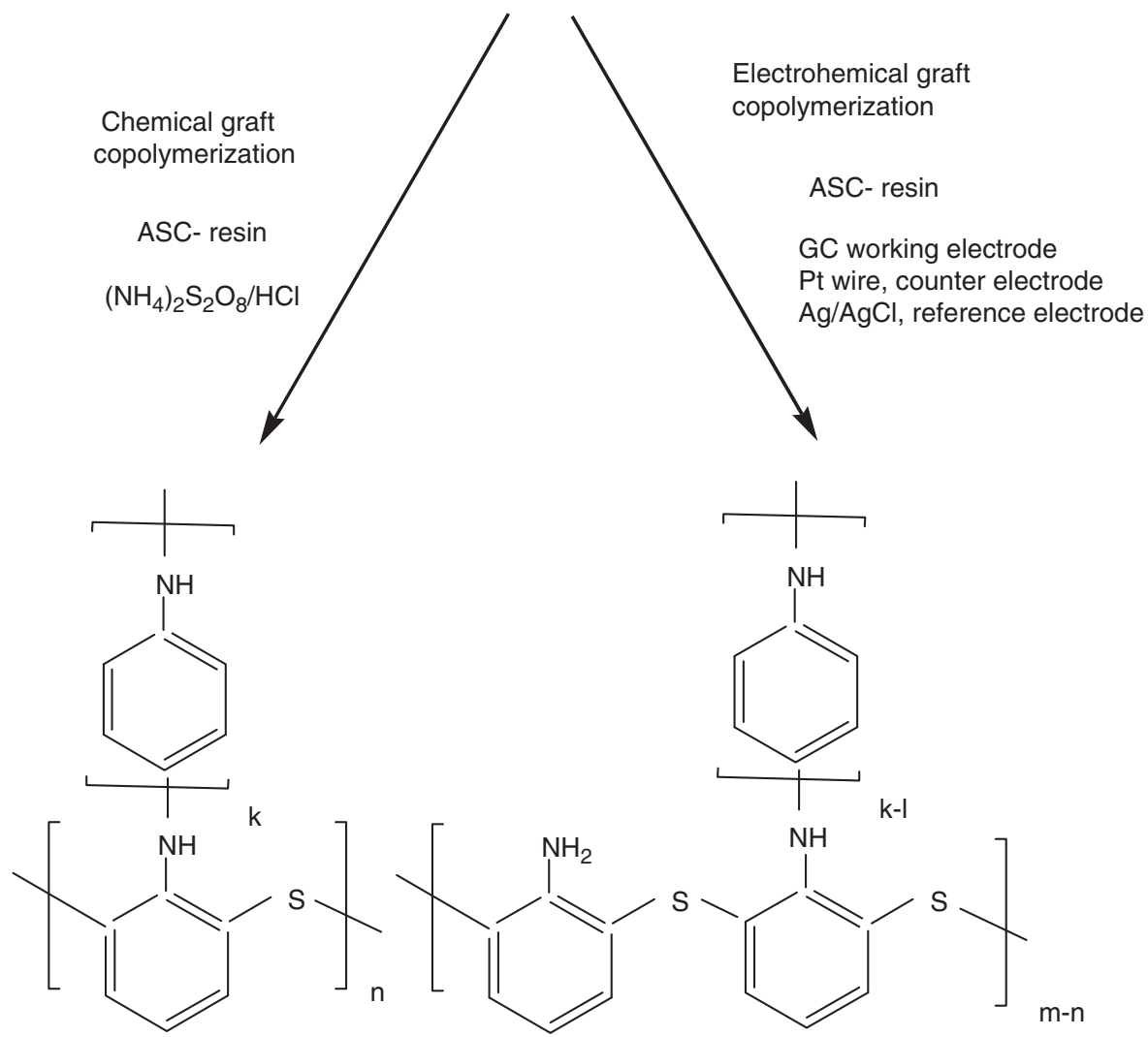

ASC

Scheme 2 Chemical and electrochemical synthesis of ASC.

can be synthesized by chemically and electrochemically oxidized ASR (Scheme 2). Aniline acts as a terminating agent in the polymerization process, and on termination of many PANi chains, the resulting graft copolymer has a branch-like structure. By controlling the initial ratio of ASR to aniline, the molecular weight of ASC can be controlled. 
Introduction

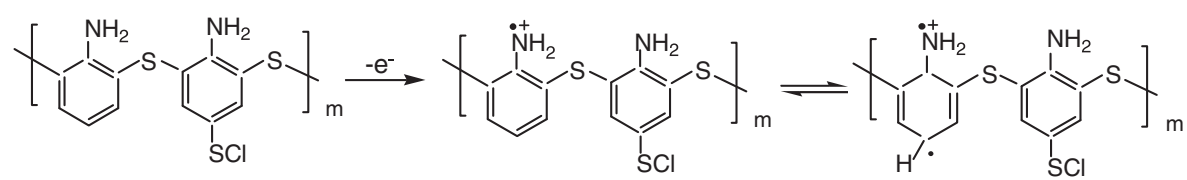

ASR

Propagation

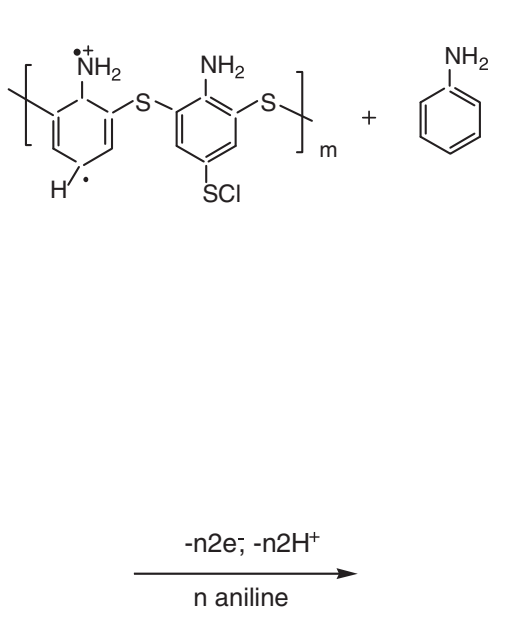

Termination

<smiles>CC(C)(C)Sc1cc(Cl)cc(Sc2cc([Tl])cc(Nc3ccc([NH-])cc3)c2Nc2ccccc2)c1N</smiles>

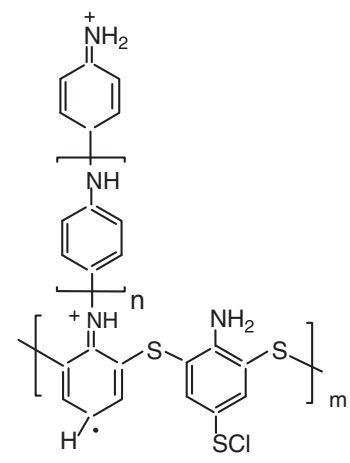

Scheme 3 Mechanism of graft copolymerization for ASC synthesis.

The mechanism for the graft copolymerization of aniline monomer was proposed as shown in Scheme 3. The graft copolymerization can be divided into three steps involving initiation, propagation and termination. The role of ASR in the rate of polymerization can be determined by the influence of ASR on all three steps of polymerization. ASR significantly influences the initiation and propagation steps. This influence is due to the growth of PANi on ASR. Therefore, it is expected that the most important role of ASR in the overall polymerization reaction will be in the initiation and propagation steps.

As a result, it is possible to change the molecular weight, solubility and conductivity of the crosslinked polymer. Under appropriate conditions, high-molecular-weight polymers can be obtained with conductivity, flexibility and solubility similar to pure PANi. The method introduced here describes a strategy for controlling the molecular weight of ASC and its physical properties. This approach may also be extended to the synthesis and modification of other polymers.

Liu and Freund ${ }^{9}$ proposed aniline formaldehyde condensation as the termination step in the same reactions, but we think aniline is the termination agent for this step in crosslinked aniline and sulfur. In any event, the influence of ASR on the rate of the overall graft copolymerization reaction can be summarized as follows:

$$
\begin{array}{ll}
\text { Initiation } & \mathrm{ASR} \stackrel{k_{1}}{\longrightarrow} \mathrm{ASR}^{\bullet+}+\mathrm{e}^{-} \\
\text {Propagation } & \mathrm{ASR}^{\bullet+}+n \mathrm{ANi} \stackrel{k_{2}}{\longrightarrow} \mathrm{ASR}-n \mathrm{ANi}^{\bullet+}\left(\mathrm{ASC}^{\bullet+}\right) \\
\text { Termination } & \mathrm{ASR}^{-n} n \mathrm{ANi}^{\bullet+}+\mathrm{ANi} \stackrel{k_{3}}{\longrightarrow} \mathrm{ASR}-(n+1) \mathrm{ANi}(\mathrm{ASC})-\mathrm{e}^{-}
\end{array}
$$

Here, $k_{1}, k_{2}$ and $k_{3}$ are corresponding reaction rate constants. 
The reaction rate for the above mechanism will then be a function of the rate of the generation of $\operatorname{ASR}^{\bullet+}$ in the initiation step,

$$
\frac{\mathrm{d}\left[\mathrm{ASR}^{\bullet+}\right]}{\mathrm{d} t}=k_{1}[\mathrm{ASR}]
$$

the rate of the generation of $\mathrm{ASC}^{\bullet+}$ in the propagation step through the reaction with monomer,

$$
\frac{\mathrm{d}\left[\mathrm{ASC}^{\bullet+}\right]}{\mathrm{d} t}=k_{2}\left[\mathrm{ASR}^{\bullet+}\right][\mathrm{ANi}]^{n}
$$

and the rate of the generation of ASC or the consumption of $\mathrm{ASC}^{\bullet+}$ in the termination step.

$$
\frac{\mathrm{d}[\mathrm{ASC}]}{\mathrm{d} t}=k_{3}\left[\mathrm{ASC}^{\bullet+}\right][\mathrm{ANi}]
$$

Assuming that the rate for the generation of $\mathrm{ASR}^{\bullet+}$ equals the rate for the consumption of $\mathrm{ASC}^{\bullet+}$ or the generation of ASC (i.e., under steady-state conditions), Equations (1) and (3) will be equal, and we know that $\left[\mathrm{ASR}^{\bullet+}\right]=\left[\mathrm{ASC}^{\bullet+}\right]$, yielding.

$$
\begin{aligned}
& k_{1}[\mathrm{ASR}]=k_{3}\left[\mathrm{ASC}^{\bullet+}\right][\mathrm{ANi}],\left[\mathrm{ASC}^{\bullet+}\right]=\left[\mathrm{ASR}^{\bullet+}\right] \\
& {\left[\mathrm{ASR}^{\bullet+}\right]=\frac{k_{1}[\mathrm{ASR}]}{k_{3}[\mathrm{ANi}]}}
\end{aligned}
$$

If the rate of polymerization is taken to be the rate of the disappearance of ASR, and if the contribution of the initiation and termination steps to the disappearance of ASR is negligible, the rate of polymerization, $R_{\mathrm{p}}$, will be equal to the rate of propagation (Equation (2)). Substituting Equation (5) into Equation (2) yields

$$
R_{\mathrm{p}}=k_{2}\left[\mathrm{ASR}^{\bullet+}\right][\mathrm{ANi}]^{n}=k_{1} k_{2} / k_{3}[\mathrm{ASR}][\mathrm{ANi}]^{n-1}
$$

\section{Molecular weight and conductivity studies}

Clearly, the ratio of ASR to aniline in the initial mixture will influence the degree of crosslinking and the space between PANi chains. These changes will in turn alter the molecular weight and the conductivity of the resulting graft copolymers. To investigate these relationships, copolymers were synthesized using different ratios of starting materials. The graft copolymerization molecular weights and conductivities for different copolymers were determined by gel permeation chromatography and a four-probe method and are summarized in Table 1. According to this table, the conductivity increases with an increasing portion of PANi. ASR is not a conductive polymer. Electric conductivity was introduced and increased by increasing the PANi ratio in the graft copolymers. Thus, ASR-PANi-3 (ASR/PANi:1/3) exhibited an electrical conductivity near to that of pure PANi.

Although PANi is not soluble in conventional organic solvents in its acid-doped form, the base form (obtained on exposure to $1 \mathrm{~m} \mathrm{NaOH}$ ) is soluble in NMP. The copolymers PANi, ASR-PANi-1, ASR-PANi-2 and ASR-PANi-3 exhibited significantly different solubilities in this same

Table 1 Starting materials and molecular weights and conductivities for different graft copolymers

\begin{tabular}{lccccc}
\hline Polymers & ASR (g) & ANi $(g)$ & $\mathrm{Mw}$ & । & Conductivity $\left(\mathrm{Scm}^{-1}\right)$ \\
\hline PANi & 0.00 & 3.0 & $1.1 \times 10^{4}$ & 2.1 & $8.1 \times 10^{-2}$ \\
ASR-PANi-1 & 0.15 & 3.0 & $6.2 \times 10^{4}$ & 4.3 & $2.7 \times 10^{-4}$ \\
ASR-PANi-2 & 0.50 & 3.0 & $3.3 \times 10^{5}$ & 5.4 & $4.3 \times 10^{-3}$ \\
ASR-PANi-3 & 1.00 & 3.0 & $1.2 \times 10^{6}$ & 7.3 & $2.2 \times 10^{-2}$ \\
\hline
\end{tabular}

Abbreviations: $\mathrm{ANi}$, aniline; ASR, aniline sulfide resin; $M w$, molecular weight; PANi, polyaniline. solvent. For example, both PANi and ASR-PANi-1 dissolve in NMP up to $\sim 8 \%$ by weight, whereas ASR-PANi- 2 and ASR-PANi-3 are only partially soluble, with ASR-PANi-3 being the least soluble. These changes in solubility can only be attributed to changes in the molecular weight of the graft copolymers, as both PANi and ASR are soluble in the NMP. Further, as ASR is soluble in the $1 \mathrm{~m} \mathrm{HCl}$ used in the washing procedure, any unreacted ASR will be removed from the final products.

\section{Electropolymerization study}

Polymer structure and morphology are greatly affected by the electrosynthetic conditions, ${ }^{13}$ such as the electrode materials, the solvent and electrolyte salts, the oxygen and water content of the system and the current density used for electropolymerization. Although a quantitative measurement of these effects has not been established, some general observations have been made. Thin films generally appear smooth, whereas thicker samples have much more uneven and textured surfaces. ${ }^{14}$ The potentials used during electropolymerization give rise to smoother films. ${ }^{15}$ Cyclic voltammetric measurements were carried out in an aqueous solution at room temperature with $0.1 \mathrm{M}$ $\mathrm{H}_{2} \mathrm{SO}_{4}$ and $1 \mathrm{M} \mathrm{HCl}(50 / 50 \mathrm{v} / \mathrm{v})$ as the supporting electrolyte. A threeelectrode system was used and included an ASR film coated with a GC working electrode $(50-60 \mu \mathrm{m})$, a Pt wire as a counter electrode and $\mathrm{Ag} / \mathrm{AgCl}$ as a reference electrode. Figure 7 shows the cyclic voltammogram of ASR under the above-mentioned conditions. The cyclic voltammogram curve shows two oxidation peaks at 0.47 and 1.16, and two reduction peaks at 0.43 and $0.94 \mathrm{~V}$, respectively. Aniline was added to the supporting electrolyte and electropolymerization was carried out for 11 cycles. Cyclic voltammetry for the preparation of ASC is shown in Figure 8. In this cyclic voltammograms in addition to

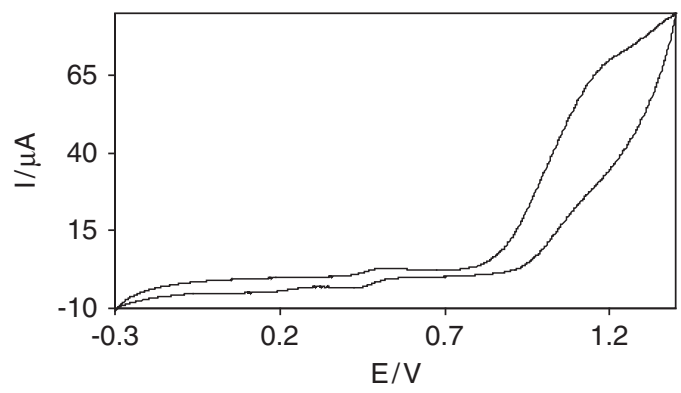

Figure 7 Cyclic voltammogram of ASR that was cast coated as a suitably thin layer $(30-35 \mu \mathrm{m})$ on a $\mathrm{GC}$ electrode in aqueous $1 \mathrm{~m} \mathrm{H}_{2} \mathrm{SO}_{4}, 0.1 \mathrm{~m} \mathrm{HCl}$ $\left(v=50 \mathrm{mV} \mathrm{s}^{-1}\right.$ ); the potential range was from 0.3 to $1.4 \mathrm{~V} \mathrm{vs} \mathrm{Ag} / \mathrm{AgCl}$.

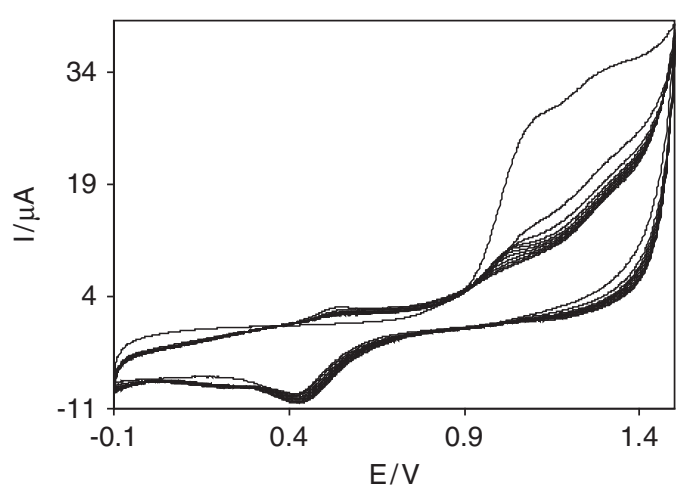

Figure 8 Cyclic voltammogram of ASC formation on a GC electrode that was cast coated by ASR in an aqueous $1 \mathrm{~m} \mathrm{H}_{2} \mathrm{SO}_{4}, 0.1 \mathrm{~m} \mathrm{HCl}$ and $0.1 \mathrm{~m}$ aniline solution $\left(v=50 \mathrm{mVs}^{-1}\right)$; the potential range was from -0.1 to $1.5 \mathrm{~V}$ vs Ag/ $\mathrm{AgCl}$ (11 cycles). 


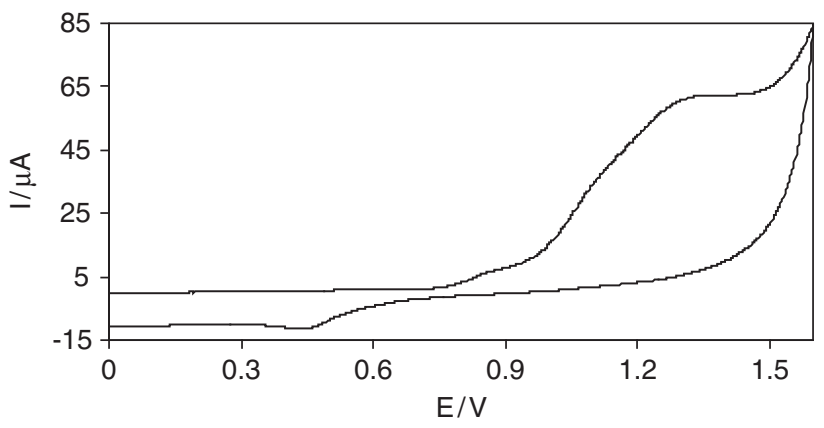

Figure 9 Cyclic voltammogram of ASC on a GC electrode in aqueous $1 \mathrm{~m} \mathrm{H}_{2} \mathrm{SO}_{4}$, $0.1 \mathrm{M} \mathrm{HCl}\left(v=50 \mathrm{mV} \mathrm{s}^{-1}\right)$; the potential range was from 0 to $1.6 \mathrm{~V}$ vs $\mathrm{Ag} / \mathrm{AgCl}$.
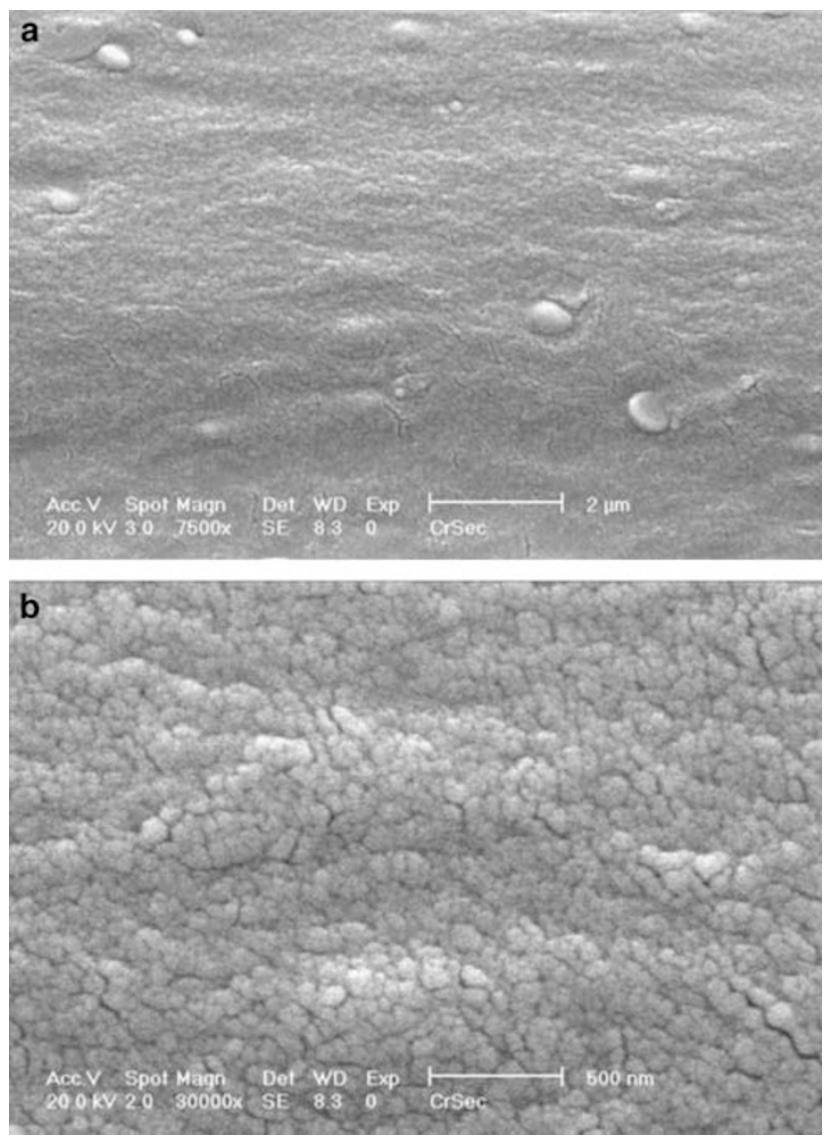

Figure 10 SEM surface images of (a) ASR and (b) ASC.

two oxidation peaks at 0.50 and 1.08 , two reduction peaks at 0.44 and $1.22 \mathrm{~V}$ can be seen. These peaks are similar to those in Figure 7 but both oxidation and reduction are decreased. Ultimately, a cyclic voltammogram of blank ASC was carried out under the above conditions and is shown in Figure 9. Accordingly, this figure shows two oxidation peaks at 0.84 and 1.28 , and one reduction peak at $0.48 \mathrm{~V}$. The reduction peak at $1.22 \mathrm{~V}$ seen in Figure 7 weakened and gradually disappeared as it is not seen in Figure 9. This influence could be due to steric interactions and crosslinks of the polymer. The thickness of the film was ca. $0.1 \mathrm{~mm}$ as determined by SEM.

\section{SEM study}

SEM shows the monotony of the kinds of phases and surfaces of the polymers. The SEM also shows that the doping/de-doping process is often accompanied by major changes in morphology, ${ }^{16}$ perhaps due to swelling caused by the insertion and removal of ions within the polymer matrix. Figures 10a and $b$ shows SEM images of ASR and ASC. As is shown in Figure 10a, the monotony and single phase of the polymers are completely clear. The PANi chain in ASC shows a sponge-like morphology, which has smooth surfaces, and no morphological characteristics could be observed.

\section{Summary}

In this paper, we chemically and electrochemically synthesized a rubbery, crosslinked, graft copolymer with ASR. This polymer has good flexibility, a rubbery behavior, an ability to form films, stability in air for years, and solubility in organic solvents such as DMSO, dimethylformamide and NMP. It is possible to control the number of crosslinked sites, the electrical conductivity, the molecular weight and the solubility, by changing the ratio of ASR to aniline during the polymerization reaction.

ASC copolymer can be synthesized by chemically oxidizing ASR in the presence of aniline. Aniline acts as a terminating agent in the polymerization process, and by terminating many PANi chains; the resulting graft copolymer has a branch-like structure. The molecular weight of ASC was controlled by controlling the initial ratio of ASR to aniline. As a result, it is possible to manipulate the molecular weight, solubility and conductivity of the crosslinked polymer. Under appropriate conditions, high-molecular-weight polymers were obtained with conductivity, flexibility and solubility similar to pure PANi. The method introduced here describes a strategy for controlling both the molecular weight of ASC and its physical properties. This approach may be extended to the synthesis and modification of other polymers.

1 Pron, A. \& Rannou, P. Processible conjugated polymers: from organic semiconductors to organic metals and superconductors. Prog. Polym. Sci. 27, 135-190 (2002).

2 Zhu, K., Wang, L., Jing, X. \& Wang, F. Poly(phenylene sulfide-tetraaniline): the soluble conducting polyaniline analogue with well-defined structures. Macromolecules 34, 8453 (2001).

3 Hosseini, S. H. \& Entezami, A. A. Chemical and electrochemical synthesis of conducting poly di-heteroaromatics from pyrrole, indole, carbazole and their mixed containing hydroxamic acid groups and studies of its metal complexes. J. Appl. Polym. Sci. 90, 63-71 (2003).

4 Leuninger, J., Wang, C., Soczka-Guth, T., Enkelmann, V., Pakula, T. \& Mullen, K. Poly(phenylene sulfide-phenyleneamine) (PPSA): the first hybrid structure of poly(phenylene sulfide) and polyaniline. Macromolecules 31, 1720 (1998).

5 Hosseini, S. H. \& Entezami, A. A. Studies of thermal and electrical conductivity behaviors of polyaniline and polypyrrole blends. Iranian Polym. J. 14, 201-209 (2005).

6 Cho, M. S., Park, S. Y., Hwang, J. Y. \& Choi, H. J. Synthesis and electrical properties of polymer composites with polyaniline nanoparticles. Mater. Sci. Eng. C 24, 15-18 (2004).

7 Hosseini, S. H. Investigation of sensing effects polystyrene graft polyaniline for cyanide compounds. J. Appl. Polym. Sci. 101, 3920-3926 (2006).

8 Ohtani, A., Abe, M., Ezoe, N., Doi, T., Miyata, T. \& Miyake, A. Synthesis and properties of high-molecular-weight soluble polyaniline and its application to the 4MB-capacity barium ferrite. Synth. Met. 1, 36963701 (1993).

9 Liu, G. \& Freund, M. S. New approach for the controlled cross-linking of polyaniline: synthesis and characterization. Macromolecules 30, 5660 (1997).

10 Ho, K. S., Hsieh, T. H., Kuo, C. W., Lee, S. W., Lin, J. J. \& Huang, Y. J. Effect of aniline formaldehyde resin on the conjugation length and structure of doped polyaniline, Spectral studies. J. Polym. Sci. A Polym. Chem. 43, 3116-3125 (2005).

11 Ho, K. S., Huang, Y. J., Kuo, C. W., Lee, S. W., Hsieh, T. H. \& Chuang, C. N. Effect of aniline-formaldehyde resin on the reduced conjugation length of doped polyaniline: thermal studies. J. Appl. Polym. Sci. 103, 2120-2128 (2006).

12 Kumar, P. A., Ray, M. \& Chakraborty, S. Hexavalent chromium removal from wastewater using aniline formaldehyde condensate coated silica gel. J. Hazard. Mater. 1-2, 24-32 (2007).

13 Pharhad Hussein, A. M. \& Kumar, A. Electrochemical synthesis and characterization of chloride doped polyaniline. Bull. Mater. Sci. 26, 329-334 (2003).

14 MacDiarmid, A. G. \& Epstein, A. J. A novel role for organic polymers. Synth. Met. 119, 27-30 (2001).

15 Bartlett, P. N. \& Simon, E. Poly(aniline)-poly(acrylate) composite films as modified electrodes for the oxidation of NADH. Phys. Chem. Chem. Phys. 2, 2599-2606 (2000).

16 Hosseini, S. H., Dabiri, M. \& Ashrafi, M. Chemical and electrochemical synthesis of conducting graft copolymer of acrylonitrile with aniline. Polym. Int. 55, 1081-1089 (2006). 